EPJ Web of Conferences 28, 12039 (2012)

DOI: $10.1051 /$ epjconf/20122812039

(C) Owned by the authors, published by EDP Sciences, 2012

\title{
Measurement of muon momentum resolution of the ATLAS detector
}

\author{
Antonio Salvucci ${ }^{1, \mathrm{a}}$ on behalf of the ATLAS Collaboration \\ Radboud University Nijmegen and Nikhef
}

\begin{abstract}
The ATLAS detector has been designed to have good muon momentum resolution up to momenta in the $\mathrm{TeV}$ range. The muon momentum resolution of the ATLAS spectrometer has been measured with $p-p$ collision data recorded in 2011. The measurement combines the di-muon mass resolution in $J / \psi \rightarrow \mu \mu$ and $Z \rightarrow \mu \mu$ decays with measurements of the alignment accuracy of the detector based on straight muon tracks, which are acquired with special runs without magnetic field in the ATLAS detector.
\end{abstract}

\section{Introduction}

The physics programme of the ATLAS Experiment [1] at the LHC includes investigations of many processes with final state muons. The ATLAS detector is equipped with a Muon Spectrometer (MS) optimized to provide a momentum measurement with a relative resolution better than $3 \%$ over a wide $p_{T}$ range and $10 \%$ at $p_{T}=1 \mathrm{TeV}$, where $p_{T}$ is the muon momentum component in the plane transverse to the beam axis. The momentum in the MS is measured from the deflection of the muon trajectory in the magnetic field generated by a system of air-core toroid coils. The MS track is reconstructed using three layers of precision drift tube (MDT) chambers in the pseudorapidity ${ }^{1}$ range $|\eta|<2$, and two layers of MDT chambers behind one layer of cathode strip chambers (CSC) for $2 \leq|\eta|<2.7$.

An additional determination of the muon momentum is provided by the Inner Detector (ID) for $|\eta|<2.5$. The ID is composed of three detectors providing coordinate measurements for track reconstruction inside a solenoidal magnetic field of $2 \mathrm{~T}$. Close to the interaction point it features a silicon pixel detector, surrounded by a silicon strip detector (SCT). In the outermost part there is a transition radiation straw tube tracker (TRT) with a coverage of $|\eta|<1.9$.

Muons entering in this analysis are reconstructed as combined muons. The underlying muon identification is described in [2] and relies on the principle that first separate tracks are measured in ID and MS before they are reconstructed as a single trajectory. This yields a higher momentum resolution than could be achieved using the individual tracks.

This paper documents the muon momentum resolution in the first pass of reconstruction of $p$ - $p$ collision data collected in 2011 corresponding to an integrated luminosity of $2.54 \mathrm{fb}^{-1}$. The first pass of reconstruction uses preliminary calibration and alignment.

\footnotetext{
${ }^{a}$ e-mail: a.salvucci@science.ru.nl

1 The pseudorapidity is $\eta=-\ln (\tan (\theta / 2))$, where $\theta$ is the polar angle with respect to the beam line.
}

\section{Parametrization of the momentum resolution as a function of $p_{T}$ and $\eta$}

The relative momentum resolution, $\sigma(p) / p$, originates from different effects $[3,4]$. The ATLAS MS is designed to provide a momentum resolution as a function of the $\eta$ and $\phi$. For a given value of $\eta$, the resolution can be parametrized as a function of $p_{T}$ :

$$
\frac{\sigma(p)}{p}=\frac{p_{0}^{M S}}{p_{T}} \oplus p_{1}^{M S} \oplus p_{2}^{M S} \cdot p_{T}
$$

where $p_{0}^{M S}, p_{1}^{M S}, p_{2}^{M S}$ are coefficients related to the energy loss in the calorimeter material, multiple scattering and intrinsic resolution terms, respectively.

For the ID, the curvature measurement depends on the track length of the muon in the active material, which is reduced close to the edge of the TRT fiducial volume. This results in a uniform response in the central part and a rapid worsening beyond this region. The approximate parametrization of the resolution is

$$
\begin{array}{ll}
\frac{\sigma(p)}{p}=p_{1}^{I D} \oplus p_{2}^{I D} \cdot p_{T} & \text { for }|\eta|<1.9 \\
\frac{\sigma(p)}{p}=p_{1}^{I D} \oplus p_{2}^{I D} \cdot p_{T} \frac{1}{\tan ^{2}(\theta)} & \text { for }|\eta|>1.9
\end{array}
$$

where $p_{1}^{I D}, p_{2}^{I D}$ are the multiple scattering and the intrinsic resolution terms, respectively.

In this study, four regions in pseudorapidity are distinguished:

- Barrel: covering $0<|\eta|<1.05$;

- Transition: covering $1.05<|\eta|<1.7$;

- End-caps: covering $1.7<|\eta|<2.0$;

- CSC/No-TRT: covering $2.0<|\eta|<2.5$.

These four regions are studied using $Z \rightarrow \mu \mu$ decays.

\section{Combined fit to the muon resolution components}

The process $Z \rightarrow \mu \mu$ is used to study the momentum resolution and to determine the corrections needed for simulation in each $\eta$ region of the detector. We use two quantities: 
i) the width of the reconstructed di-muon invariant mass peak at the $\mathrm{Z}$ pole, which is a convolution of the natural width of the $\mathrm{Z}$ boson and the muon momentum resolution;

ii) the difference between the independent momentum measurements of the ID and MS for combined muons, which is sensitive to the quadratic sum of the ID and MS momentum resolutions. This difference is weighted by the muon electric charge $\left(q / p_{T}^{I D}-q / p_{T}^{M S}\right)$ : this disentangles systematic effects of the curvature due to local misalignements from the overall intrinsic resolution, reducing the bias on the estimation of the resolution and correction parameters.

\subsection{Global fit procedure}

Using the previous inputs, the measurements of the MS and ID momentum resolution are obtained using a Monte Carlo template technique, based on a "global" fit procedure with:

i) a template fit to reconstructed $Z$ lineshape:

we allow for momentum resolution smearing in the fit to the $\mathrm{Z}$ lineshape obtained from MS and ID tracks and a combined fit with events with muons in different detector regions. In this case we are sensitive to $\sigma_{\text {mult.scatt. }} \oplus \sigma_{\text {intrinsic }}$

ii) the above, plus template fit to $\left(q / p_{T}^{I D}-q / p_{T}^{M S}\right)$ distribution: we allow for momentum resolution smearing in the fit, that compute several bins of $p_{T}$, keeping regions separated. This case is sensitive to $\sigma_{I D} \oplus \sigma_{M S}$

iii) external constraints on MS alignment and multiple scattering in ID and MS (see 3.2)

Concerning the smearing, for both the MS and the ID, the transformation of $p_{T}$ is

$$
p_{T}^{\prime}=p_{T}\left(1+g \Delta p_{1}^{I D, M S}+g \Delta p_{2}^{I D, M S} p_{T}\right)
$$

where $p_{T}^{\prime}$ indicates the simulated muon $p_{T}$ after applying the correction $\Delta p_{i}^{I D, M S}$, while $g$ is a normally distributed random number with mean 0 and width 1 .

\subsection{External constraints to the combined fit}

In the fitting procedure, additional knowledge is introduced from independent studies, both for the ID and the MS. This reduces the correlation among the multiple scattering and the detector resolution terms in the fit, resulting in smaller uncertainties on the fitted parameters.

For the ID, the correction to the multiple scattering term in the ID, $\Delta p_{1}^{I D}$, is fixed to a value of zero, due to a knowledge of the ID material of $0.05 \%$.

For the MS, the multiple scattering term, $\Delta p_{1}^{M S}$, is a free parameter of the fit. The energy loss of muons is mainly concentrated in the calorimeter and has been measured in [6]. Its contribution to the overall MS resolution in the $p_{T}$ range from $20 \mathrm{GeV}$ to $100 \mathrm{GeV}$ is negligible and so no additional contribution for the energy loss, $\Delta p_{0}^{M S}$, is included. For the intrinsic resolution term, $\Delta p_{2}^{M S}$, the best estimate of the alignment accuracy is applied. This is the result of studies from samples of straight tracks obtained in periods of collision data taken with no magnetic field in the muon system. The constraints on $\Delta p_{2}^{M S}$ are reported on Table 1 .
Table 1. Constraints on the intrinsic resolution term

\begin{tabular}{cc}
\hline$\eta$ region & Constraint on $\Delta p_{2}^{M S}\left(\mathrm{TeV}^{-1}\right)$ \\
\hline barrel & $0.143 \pm 0.030$ \\
transition & $0.312 \pm 0.050$ \\
end-caps & $0.200 \pm 0.050$ \\
CSC/No-TRT & $0.408 \pm 0.050$ \\
\hline
\end{tabular}

\section{Combined fit results}

The constraints on the $\Delta p_{i}$ parameters are applied in the combined fit by adding a penalty term $\sum_{i}\left(\frac{\Delta p_{i}-a_{i}}{\sigma_{a_{i}}}\right)^{2}$ to the total $\chi^{2}$ being minimized, where $a_{i}$ is the expectation value and $\sigma_{a_{i}}$ the associated uncertainty for each of the constrained $\Delta p_{i}$ parameters. The fitted corrections parameters are provided in Table 2 together with their statistical and systematic uncertainties. Figure 1 shows an example of $\mathrm{Z}$

Table 2. Set of corrections to be applied on the $p_{T}$ parametrization of the simulated resolution in the MS and ID to reproduce the one in data. Systematic errors on the transition region are due to a different definition of the region itself $(1.2<|\eta|<1.7)$

\begin{tabular}{ccc}
\hline$\eta$ region & $\Delta p_{1}^{M S}(\%)$ & $\Delta p_{2}^{M S}\left(\mathrm{TeV}^{-1}\right)$ \\
\hline barrel & $1.80 \pm 0.05$ & $0.095 \pm 0.016$ \\
transition & $3.17 \pm 0.15 \pm 0.22$ & $0.250 \pm 0.026 \pm 0.067$ \\
end-caps & $1.23 \pm 0.11$ & $0.169 \pm 0.069$ \\
CSC/No-TRT & $0.52 \pm 0.58$ & $0.453 \pm 0.028$ \\
\hline$\eta$ region & $\Delta p_{1}^{I D}(\%)$ & $\Delta p_{2}^{I D}\left(\mathrm{TeV}^{-1}\right)$ \\
\hline barrel & 0 & $0.283 \pm 0.011$ \\
transition & 0 & $0.736 \pm 0.022 \pm 0.567$ \\
end-caps & 0 & $0.871 \pm 0.017$ \\
CSC/No-TRT & 0 & $0.050 \pm 0.001$ \\
\hline
\end{tabular}
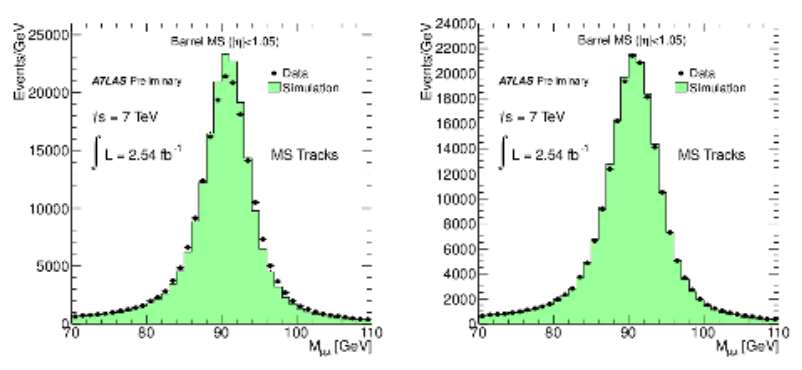

Fig. 1. Examples of $\mathrm{Z}$ lineshape fit for MS tracks in the barrel region, before (on the left) and after fit (on the right).

lineshape fit for MS tracks in the barrel region, before (left) and after (right) the fit.

The values of the correction parameters quantify the increase in momentum resolution in data when compared to simulation. The full parametrization of the experimental momentum resolution is obtained by quadratically adding the uncorrected simulated resolution terms of equations 13 and the corresponding parameters from Table 2 . The results for the full parametrization are listed in Table 3. 
Table 3. Resolution parametrization as defined in equations 1-3 in the MS and ID.

\begin{tabular}{cccc}
\hline$\eta$ region & $p_{0}^{M S}(\mathrm{TeV})$ & $p_{1}^{M S}(\%)$ & $p_{2}^{M S}\left(\mathrm{TeV}^{-1}\right)$ \\
\hline barrel & $0.25 \pm 0.01$ & $3.27 \pm 0.05$ & $0.168 \pm 0.016$ \\
transition & 0 & $6.49 \pm 0.26$ & $0.336 \pm 0.072$ \\
end-caps & 0 & $3.79 \pm 0.11$ & $0.196 \pm 0.069$ \\
CSC/No-TRT & $0.15 \pm 0.01$ & $2.82 \pm 0.58$ & $0.469 \pm 0.028$ \\
\hline$\eta$ region & $p_{0}^{I D}(\mathrm{TeV})$ & $p_{1}^{I D}(\%)$ & $p_{2}^{I D}\left(\mathrm{TeV}^{-1}\right)$ \\
\hline barrel & n.a & $1.55 \pm 0.01$ & $0.417 \pm 0.011$ \\
transition & n.a & $2.55 \pm 0.01$ & $0.801 \pm 0.567$ \\
end-caps & n.a & $3.32 \pm 0.02$ & $0.985 \pm 0.019$ \\
CSC/No-TRT & n.a & $4.86 \pm 0.22$ & $0.069 \pm 0.003$ \\
\hline
\end{tabular}

\section{Measured resolutions as a function of $p_{T}$}

The parametrized resolution as a function of $p_{T}$ for the barrel region, obtained using the values of the parameters from the combined fits, are shown in figure 2 for the MS and the ID. The resolution curves for experimental data (in blue) are compared to those from uncorrected parameters obtained for the simulation (in red). To indicate the
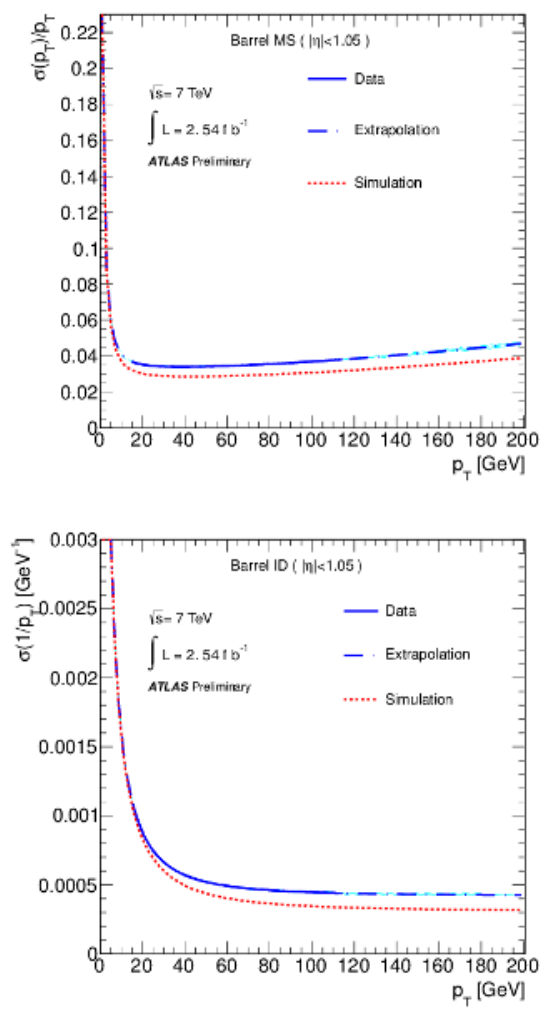

Fig. 2. Resolution curve from the fitted parameter values on the MS (top) and the ID (bottom) in collision data and simulation as a function of the muon $p_{T}$, for the barrel region. The solid blue line shows determinations based on data, the dashed blue line shows the extrapolation to $p_{T}$ range not accessible in this analysis and the dashed red line shows the determinations from simulation.

goodness of the simulation correction provided in section 4 , figure 3 shows the distribution of the di-muon invariant mass in the $\mathrm{Z}$ region after applying the corrections, for $\mathrm{MS}$, ID and combined tracks.
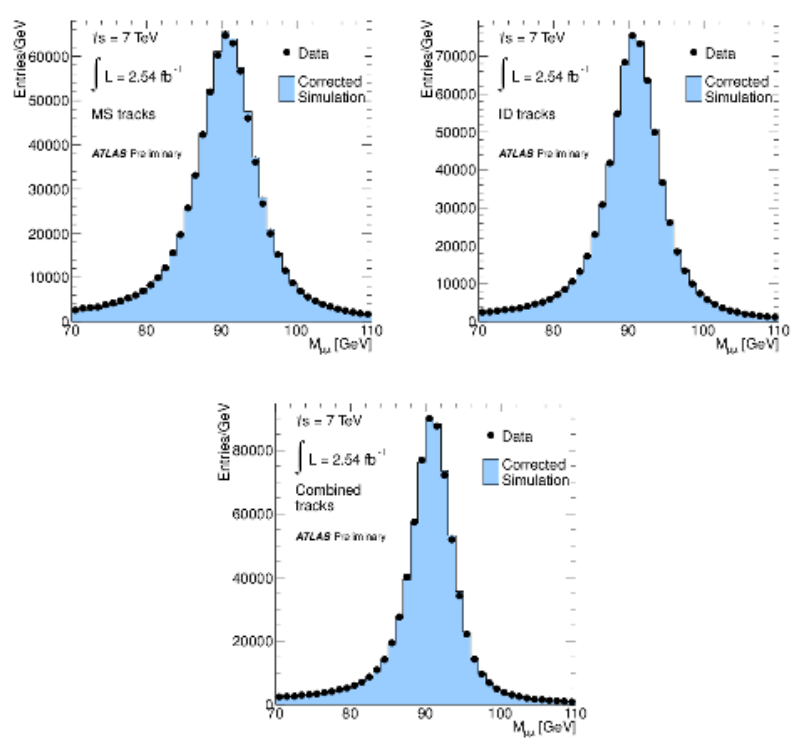

Fig. 3. Di-muon invariant mass comparison in the $\mathrm{Z}$ boson mass range between collision data (dots) and simulation (full histogram), after correcting the simulated muon $p_{T}$, in the full range of $\eta$. From top left to bottom: MS, ID and combined measureaments are shown.

\section{Conclusions}

A determination of the muon momentum resolution is presented for the integrated luminosity of $2.54 \mathrm{fb}^{-1}$ collision data collected in 2011 with the ATLAS detector. $Z \rightarrow \mu \mu$ decays have been used to evaluate the resolution as a function of the muon $p_{T}$ and $\eta$, for both the MS and the ID. The momentum resolutions were measured on the experimental data and compared with the simulation. Results obtained present an improvement of the resolution and the alignment with respect to those obtained with 2010 data [7].

\section{References}

1. ATLAS Collaboration, The ATLAS Experiment at the CERN Large Hadron Collider, JINST 3 (2008) S08003

2. ATLAS Collaboration, Determination of the mиоn reconstruction efficiency in ATLAS at the $Z$ resonance in $p$ - $p$ collisions at $\sqrt{s}=7 \mathrm{TeV}$, ATLAS-CONF-2011-008 (2011).

3. ATLAS Collaboration, Commissioning of the ATLAS Muon Spectrometer with Cosmic Rays, Eur.Phys.J. C 70, (2010) 875.

4. ATLAS Collaboration, The ATLAS Inner Detector commissioning and calibration, Eur.Phys.J. C 70, (2010) 787.

5. ATLAS Collaboration, Study of the Material Budget in the ATLAS Inner Detector with $K_{S}^{0}$ decays in collision data at $\sqrt{s}=900 \mathrm{GeV}$, ATLAS-CONF-2010-019 (2010).

6. ATLAS Collaboration, Studies of the perfomance of the ATLAS detector using cosmic-ray muons Eur.Phys.J. C71 (2011) 1593.

7. ATLAS Collaboration, ATLAS Muon Momentum Resolution in the First Pass Reconstruction of the 2010 p-p Collision Data at $\sqrt{s}=7 \mathrm{TeV}$, ATLAS-CONF-2011-046 (2011). 